\title{
Índices de seleção para bovinos da raça Nelore participantes de provas de ganho em peso em confinamento
}

\author{
Selection indices for Nellore beef cattle from performance test weight in confinement
}

\author{
MARQUES, Ednira Gleida ${ }^{1 *}$; MAGNABOSCO, Claudio Ulhoa ${ }^{2}$; LOPES, Fernando Brito ${ }^{2}$ \\ ${ }^{1}$ Universidade Federal de Goiás, Escola de Medicina Veterinária e Zootecnia, Programa de Pós- \\ Graduação em Ciência Animal, Goiânia, Goiás, Brasil. \\ ${ }^{2}$ Embrapa Cerrados, Goiânia, Goiás, Brasil. \\ *Endereço para correspondência: gleidamarques@ hotmail.com
}

RESUMO

Objetivou-se com este estudo avaliar a evolução das características mensuradas nas provas de ganho em peso, verificar os ponderadores dos índices empíricos utilizados atualmente e sugerir índices de seleção para classificar os animais participantes dos testes de desempenho. Os animais participantes das provas de ganho em peso em regime de confinamento são selecionados de acordo com todas as características de importância econômica, mas o faz de maneira subjetiva. Tais índices têm sido propostos em programas de melhoramento animal como método de selecionar caracteres simultaneamente. As informações analisadas, no presente estudo, foram obtidas nos anos de 2000 a 2010, em bovinos da raça Nelore, provenientes de 392 provas de ganho em peso confinadas, oficializadas pela Associação Brasileira dos Criadores de Zebu - ABCZ. Foram avaliadas as características de ganho em peso médio diário, peso final ajustado aos 426 dias, perímetro escrotal e EPMURAS. Os valores genéticos dos animais para cada característica foi predito por meio do programa MTDFREML. Após a composição dos índices de seleção, foi elaborada uma simulação em que os parâmetros genéticos foram aproveitados do banco de dados original pelo valor genético estimado. Os resultados deste trabalho permitem concluir que ao utilizar os índices, cujo objetivo de seleção é o peso final, maior será o ganho genético.

Palavras-chave: ganho genético, melhoramento genético animal, progresso genético, zebu.

\section{SUMMARY}

Despite many subjective criteria are still adopted, feedlot bovines are currently selected by weight gain tests according to varied economic traits. Considering this situation, selection indexes are becoming a possibility to select multiple traits simultaneously in genetic breeding programs. Thus, this study was carried out to investigate the development of traits measured in weight gain evaluations, to verify empiric index pondered and to suggest selection indexes to classify animals in performance tests. Data analyzed in this study was obtained from 392 weight gain trails in Nellore cattle in feedlot, during 2000 and 2010. The information was supplied by the Brazilian Association of Zebu Breeders - ABCZ. Average daily weight gain, final body weight adjusted to 426 days, scrotal perimeter and EPMURAS were evaluated. The animal predicted breeding value for each trait was carried out using MTDFREML software. Following the selection index composition, a simulation was undertaken using genetic parameters obtained from an original database from the predicted genetic value. In conclusion, by the use of indexes aiming at selection based on final weight it is possible to obtain greater genetic gain.

Keyword: animal breeding, genetic gain, genetic progress, zebu. 


\section{INTRODUÇÃOO}

A seleção em bovinos de corte tem por base, em grande parte, características produtivas (PEDROSA et al., 2010). Entretanto, considerar uma única característica não é suficiente para representar o mérito genético $\mathrm{e}$ econômico de uma população. A escolha de critérios de seleção corretos e a maneira como estes devem ser ponderados são as decisões mais importantes a serem tomadas pelo produtor (QUEIROZ et al., 2005; BETT et al., 2007).

Um dos principais obstáculos para o cálculo dos objetivos de seleção é a correta determinação dos valores econômicos das características (HAZEL, 1943; KRUPOVA et al., 2008; CHEN et al., 2009; KRUPOVA et al., 2009; ZHANG et al., 2009), os quais são essenciais à predição da rentabilidade dos programas de seleção (TOZER \& STOKEST, 2002; WOLFOVÁ et al., 2007a; WOLFOVÁ et al., 2007b; WOLFOVÁ et al., 2007c; LÔBO et al., 2010).

Muitas vezes é difícil definir os critérios de seleção (MARTINS et al., 2003; KRUPOVA et al., 2008; KRUPOVA et al., 2009), pois a produção animal varia entre sistemas e regiões. Essas diferenças são intrínsecas ao manejo aplicado, ou mesmo, resultantes da aplicação de novas tecnologias as quais nem sempre estão acessíveis a todos os criadores. Portanto, como forma de contornar essa dificuldade, é importante definir o objetivo de seleção e elaborar um índice econômico de seleção (MAGHSOUDI et al., 2009; TABBAA \& AL-ATIYAT, 2009).

A escolha de múltiplas características a partir de índices de seleção é a maneira mais rápida e eficiente de melhorar o valor genético agregado, pois utiliza uma grande quantidade de informação de várias características para produzir um valor único (CUNNINGHAM \& TAUEBERT, 2009; LAMBE et a., 2008; QUEIROZ et al., 2005; TABBAA \& ALATIYAT, 2009).

Dessa forma, para que a pecuária seja mais eficiente, faz-se necessária a utilização racional dos recursos genéticos e ambientais, de forma a maximizar o retorno líquido da atividade exercida. A definição dos objetivos de seleção deve ser o primeiro passo na elaboração de um programa de melhoramento genético (BETT et al., 2007; DUBEUF \& BOYAZOGLU, 2009; LÔBO et al., 2010).

Objetivou-se com este estudo avaliar a evolução das características mensuradas nas provas de ganho em peso, verificar os ponderadores dos índices empíricos utilizados atualmente e sugerir índices de seleção para classificação de bovinos da raça Nelore participantes dos testes de desempenho em confinamento.

\section{MATERIAL E MÉTODOS}

Para comparação entre o índice de seleção empírico (JOSAHKIAN et al., 2009), atualmente utilizado em provas de ganho em peso, e os índices de seleção adaptados de Hazel et al. (1943), as análises foram realizadas em duas etapas: realização de análise bicaráter, com a finalidade de se estimar os componentes de (co)variâncias e parâmetros genéticos, essenciais para a elaboração dos índices de seleção; e análise unicaráter, com finalidade de predizer o valor genético dos animais, oriundos de um rebanho simulado, para comparação do progresso genético obtido por meio dos três índices avaliados. 
As informações analisadas, no presente estudo, necessárias para elaboração da matriz de (co)variâncias genéticas e fenotípicas, a qual foi utilizada na elaboração dos índices de seleção (HAZEL et al, 1943), foram obtidas no período de 2000 a 2010 oriundas de um banco de dados de bovinos da raça Nelore, provenientes de 392 provas de ganho em peso em regime de confinamento, oficializadas pela Associação Brasileira dos Criadores de Zebu - ABCZ. Foram estudadas as características de peso final ajustado aos 426 dias (PF), ganho médio diário (GMD), perímetro escrotal (PE) e EPMURAS (estrutura, precocidade, musculosidade, umbigo, padrão racial, aprumos e característica sexual).

Foram cedidos, pela $\mathrm{ABCZ}$ dois conjuntos de dados: um arquivo de genealogia e outro conjunto de dados, que possuía os registros de desempenho e informações das provas de ganho em peso em regime de confinamento (arquivos de dados com 11.260 animais). $\mathrm{O}$ arquivo de dados foi inicialmente analisado com o objetivo de verificar a existência de outliers, provenientes de informações coletadas e/ou informadas de forma inconsistente. Deste, foi criado um novo arquivo, composto de registros de 9.970 animais. $\mathrm{O}$ arquivo de pedigree, inicialmente fornecido, foi formatado para manter apenas as genealogias relacionadas ao novo arquivo de dados. Dessa forma, o arquivo de pedigree é constituído de 40.415 animais. Para tanto, foram considerados todos os animais até a nona geração de parentesco. Para edição e análise dos dados foi utilizado o programa computacional SAS® (SAS INSTITUTE, 2004).

Os grupos de contemporâneos (GC) foram formados por animais pertencentes à mesma prova de ganho em peso, ou seja, animais do mesmo sexo, provenientes do mesmo ano e estação de nascimento e que receberam o mesmo manejo nutricional. Foram utilizados dados de 11 anos e descartados os registros que contemplavam avaliações de tipo obtidas por outra metodologia não mais utilizada pela $\mathrm{ABCZ}$, ou seja, foram analisados registros de animais, cuja avaliação de tipo foi obtida pela metodologia EPMURAS.

Todas as variáveis foram verificadas em detalhes por meio do procedimento PROC UNIVARIATE a fim de se testar a distribuição dos dados. Todas as análises foram realizadas através do programa computacional Statistical Analysis System (SAS INSTITUTE, 2004). A estatística descritiva que continha número de animais, média, desvio padrão $\mathrm{e}$ coeficiente de variação para as características estudadas, é representada pela na Tabela 1.

Tabela 1. Número de animais, média, desvio padrão e coeficiente de variação das características estudadas

\begin{tabular}{lcccc}
\hline Características & $\begin{array}{c}\mathrm{N}^{\mathbf{o}} \text { de } \\
\text { animais }\end{array}$ & Média & $\begin{array}{c}\text { Desvio- } \\
\text { padrão }\end{array}$ & $\begin{array}{c}\text { Coeficiente de } \\
\text { variação }(\%)\end{array}$ \\
\hline Peso final $(\mathrm{kg})$ & 9.970 & 375,27 & 58,19 & 15,51 \\
Ganho médio diário $(\mathrm{kg})$ & 9769 & 0,89 & 0,25 & 28,64 \\
EPMURAS & 6052 & 25 & 4,21 & 16,82 \\
Perímetro escrotal $(\mathrm{cm})$ & 3155 & 25,86 & 3,43 & 12,29 \\
\hline
\end{tabular}

EPMURAS: avaliação de tipo. 
Na primeira etapa das análises genéticoquantitativas, as informações oriundas das provas de ganho em peso, foram analisadas por meio de procedimentos bicaracterísticos. O modelo estatístico incluiu os efeitos fixos dos grupos de contemporâneos, o efeito aleatório genético aditivo direto e os efeitos residuais. As análises das variáveis em estudo foram realizadas segundo modelo animal:

$$
\mathrm{y}=\mathrm{X} \beta+\mathrm{Za}+\mathrm{e}
$$

em que:

$y$ : vetor de observações (peso final, ganho médio diário, avaliação de tipo e perímetro escrotal);

$\boldsymbol{\beta}$ : vetor do efeito fixo (grupo de contemporâneo); $\boldsymbol{a}$ : vetor do efeito genético aditivo;

$\boldsymbol{X}$ : matriz de incidência que associa $\beta$ comy;

$\boldsymbol{Z}$ é $a$ matriz de incidência do efeito genético aditivo;

$\boldsymbol{e}$ : vetor residual.

Foram realizadas análises unicaracterísticas com a finalidade de predizer os valores genéticos (parâmetros genéticos, termo correto) para peso final, ganho médio diário, avaliação de tipo e perímetro escrotal. Já as análises bicaracterísticas foram realizadas para se estimar as correlações fenotípicas e genéticas entre as características em estudo. Haja vista que as estimativas de herdabilidades não apresentaram diferenças, quando estimadas por meio das análises unicaráter e bicaráter, optouse por apresentar as estimativas de parâmetros genéticos e componentes de (co)variância obtidos por meio das análises bicaráter.

Os componentes de (co)variância necessários para obtenção dos parâmetros genéticos, foram estimados pela metodologia Restricted Maximum Likelihood (REML) sob modelo animal, por meio do aplicativo Multiple trait
Derivate-Free Restricted Maximum Likelihood (MTDFREML) desenvolvido por Boldman et al. (1995). O critério de convergência considerado para a implementação com MTDFREML foi de $10^{-9}$. Cabe lembrar que, a cada convergência, o programa foi reiniciado seis vezes com os parâmetros genéticos obtidos da análise anterior.

A segunda etapa das análises consistiu na elaboração dos índices de seleção e na simulação de rebanhos para que os índices de seleção pudessem ser testados e comparados com o índice empírico. Uma vez que não se conhecia o valor econômico das características em estudo, foram calculados índices de seleção para os objetivos de seleção peso final e ganho médio diário. Estes foram escolhidos devido à relevância econômica nas provas de ganho em peso e aos valores de suas herdabilidades. Assim, os vetores de valores econômicos multiplicados pela matriz de (co)variâncias genéticas utilizados foram $a_{1}=[1,0,0,0$,$] e a_{2}=[0$, $1,0,0]$, respectivamente para os índices com objetivo de seleção para peso final (PF) e ganho em peso médio diário (GMD).

Os valores genéticos e fenotípicos variam em função de cada característica em estudo e o valor agregado pode ser obtido pelo somatório dos vários genótipos existentes e suas relações com o valor econômico. Esse genótipo agregado foi representado por:

$$
\mathrm{H}=\mathrm{a}_{1} \mathrm{G}_{1}+\mathrm{a}_{2} \mathrm{G}_{2}+\ldots+\mathrm{a}_{\mathrm{n}} \mathrm{G}_{\mathrm{n}} \text {. }
$$

$\mathrm{O}$ mérito genético agregado foi então definido como: $H=\sum a_{i} G_{i}$, em que $\boldsymbol{H}$ é uma função linear dos valores genéticos para cada uma das características de interesse $\left(\boldsymbol{G}_{\boldsymbol{i}}\right)$ ponderadas pelo seu valor econômico relativo $\left(\boldsymbol{a}_{\boldsymbol{i}}\right)$. Este autor definiu os valores econômicos como "a quantidade esperada de aumento no lucro por cada unidade de melhoramento na característica $i$ ". Assim, foi introduzido o 
conceito de genótipo agregado ou do objetivo de seleção $(\boldsymbol{H})$, definido como uma função linear dos valores genéticos ponderados pelos valores econômicos de cada característica (HAZEL, 1943).

Uma vez definidos os objetivos e escolhidos os critérios de seleção, foram calculados os índices de seleção, cujas informações provieram do banco de dados analisados na primeira parte do trabalho, cujos resultados estão apresentados na tabela 2. O índice de seleção foi definido por:

$I=b{ }_{1} X_{1}+b_{2} X_{2}+\ldots+b \quad{ }_{n} X_{n}$ em que, $X_{1}, X_{2}, \ldots, X_{n}$ representam o desempenho das diversas características e $b_{1}, b_{2}, \ldots, b_{n}$ são os coeficientes de regressão obtidos de modo que a correlação $\left(\boldsymbol{r}_{I H}\right)$ entre o índice e o objetivo de seleção seja maximizada (HAZEL, 1943).

As matrizes de (co)variâncias fenotípicas e genética aditiva, utilizadas na simulação dos dados, foram obtidas por meio das correlações e variâncias genéticas e fenotípicas, estimadas em análises bicaráter para as características avaliadas nas provas de ganho em peso (Tabela 2).

Tabela 2. Estimativas de correlação genética (acima da diagonal), fenotípicas (abaixo da diagonal), herdabilidade (diagonal) e variância genética aditiva e fenotípica para PF, GMD, EPMURAS e PE

\begin{tabular}{lcccc}
\hline Características & PF & GMD & EPMURAS & PE \\
\hline PF & 0,60 & 0,24 & 0,58 & 0,73 \\
GMD & 0,12 & 0,55 & 0,23 & $-0,11$ \\
EPMURAS & 0,53 & 0,11 & 0,63 & $-0,08$ \\
PE & 0,62 & 0,11 & 0,15 & 0,55 \\
\hline \multicolumn{5}{c}{ Componentes de variância genética aditiva e fenotípica } \\
\hline$\sigma_{a}^{2}$ & 1231,40 & 0,21 & 16,07 & 14,12 \\
$\sigma_{p}^{2}$ & 2059,20 & 0,37 & 25,37 & 25,84 \\
\hline$\sigma_{a}^{2}=$ variância genética aditiva; $\sigma_{p}^{2}=$ variância fenotípica; PF = peso final; GMD = ganho médio \\
diário; EPMURAS = avaliação de tipo; e PE = perímetro escrotal.
\end{tabular}

A meta foi estimar os coeficientes do índice de seleção, $\boldsymbol{b}_{\boldsymbol{n}}$, de modo que a seleção de indivíduos, baseada nos valores de seus índices, $\boldsymbol{I}$, maximize a resposta no genótipo agregado, $\boldsymbol{H}$. A estimativa dos ponderadores dos índices foi calculada por meio do seguinte modelo, $\mathrm{b}=\mathrm{P}^{-1} \mathrm{Ga}$, em que, $\boldsymbol{P}$ é matriz $n x n$ de (co)variâncias fenotípicas das características de $\boldsymbol{I}$; e, $\boldsymbol{G}$ é a matriz mxm de (co)variâncias genéticas aditivas entre as características incluídas em $\boldsymbol{H}$ e em $\boldsymbol{I} ;$ e, $\boldsymbol{a}$ é vetor de valores econômicos.

Ao se aplicar a equação geral de resposta à seleção $\left(\boldsymbol{S}_{\boldsymbol{H}}\right)$, baseada em $\boldsymbol{I}$ para melhorar $\boldsymbol{H}$, pode-se obter a predição da superioridade genética para o genótipo agregado, $\boldsymbol{H}$, em relação à seleção praticada sobre o índice, $\boldsymbol{I}$. Assim, a resposta à seleção foi calculada por meio da seguinte fórmula, $S_{H}=\left(i \times r_{H I} \times \sigma_{H}\right) / L$, em que, $i$ é a intensidade de seleção praticada, $\boldsymbol{r}_{\boldsymbol{H}}$ é a 
acurácia da seleção baseada em $\boldsymbol{I}$ e $\sigma_{H}$ é o desvio padrão de $\boldsymbol{H}$. Foi considerada uma intensidade de seleção de 1,28 com retenção de $10 \%$ dos machos e $50 \%$ das fêmeas, e o intervalo médio de gerações $(\boldsymbol{L})$ de 6,5 anos. Para a solução dos sistemas lineares e a obtenção dos coeficientes de regressão dos índices e demais parâmetros analisados, utilizouse o procedimento Interactive Matrix Language (IML) do aplicativo SAS (SAS INSTITUTE, 2004).

Após, a criação dos índices de seleção foi realizada uma simulação, cujos parâmetros genéticos e fenotípicos (Tabela 2) foram obtidos por meio do banco de dados original. O rebanho foi composto inicialmente por 1000 fêmeas, acasaladas aleatoriamente com 50 touros, não selecionados e não aparentados. Foram realizados descartes dos machos com menor valor genético e seleção dos filhos com maior valor genético. Todos os animais selecionados e mantidos no rebanho foram classificados por meio dos índices empíricos e de seleção. Os reprodutores foram substituídos após cinco anos de uso. Cinco gerações foram simuladas, a totalizar 5 mil registros.

A seleção dos melhores animais que permaneceram no rebanho foi realizada com base no mérito genético total, obtido mediante os índices empíricos e índices de seleção para os objetivos: peso final (PF) e ganho em peso médio diário (GMD). Dessa forma, foram simulados três rebanhos: o primeiro rebanho foi simulado a considerar a seleção dos melhores animais pelos índices cujo objetivo foi peso final; o segundo rebanho foi simulado a considerar a seleção dos melhores animais pelos índices cujo objetivo foi ganho médio diário; o terceiro rebanho foi simulado a considerar a seleção dos melhores animais pelos índices empíricos.
As observações referentes ao ganho médio diário (GMD), peso final (PF), avaliação de tipo (EPMURAS) e perímetro escrotal (PE) foram simuladas, por meio de análise multivariadas, com base no seguinte modelo matemático, $Y_{i j}=\mu+A_{i}+e_{i j}$, em que $\mathbf{Y}_{i j}$ é o peso final, ganho médio diário, avaliação de tipo e perímetro escrotal; $\mathbf{A}_{\mathbf{i}}$ é o efeito genético aditivo; $\mathbf{e}_{\mathrm{ij}}$ é erro aleatório, peculiar a cada observação.

Como todas as características foram simuladas simultaneamente, por meio das matrizes de (co)variância genética aditiva direta e residual (Tabela 2), os efeitos genéticos aditivos direto foram considerados geneticamente correlacionados. Esse processo sustentase na decomposição de Cholesky da matriz $\mathbf{G}$ de (co)variâncias genéticas, a qual pode ser expressa pelo produto de uma matriz vezes sua transposta, isto é:

$$
G=\left(\begin{array}{cc}
\sigma_{a 1}^{2} & \sigma_{a 1 a 2} \\
\sigma_{a 1 a 2} & \sigma_{a 2}^{2}
\end{array}\right)=\left(\begin{array}{cc}
C_{11} & 0 \\
C_{12} & C_{22}
\end{array}\right) \times\left(\begin{array}{cc}
C_{11} & C_{12} \\
0 & C_{22}
\end{array}\right)
$$

em que $\mathbf{C}$ é uma matriz triangular inferior com os valores $\mathbf{C}_{\mathbf{1 1}}, \mathbf{C}_{\mathbf{1 2}}$ e $\mathbf{C}_{\mathbf{2 2}}$ correspondentes aos componentes $\sigma_{\mathrm{a} 1}^{2}$, $\sigma_{\mathrm{a} 1 \mathrm{a} 2}$ e $\sigma_{\mathrm{a} 2}^{2}$, respectivamente.

As gerações subsequentes foram simuladas e revelaram que o valor de um animal $\left(\mathbf{g}_{\mathbf{n}}\right)$ é igual ao valor médio de seus pais $\left(\mathbf{g}_{\mathbf{m}}\right.$ e $\left.\mathbf{g}_{\mathbf{p}}\right)$ mais o efeito de segregação Mendeliana, multiplicado por um valor aleatório de distribuição normal com média zero e variância um, conforme descrito por Clément et al. (2001): $g_{n}=\left(\left(g_{m}+g_{p}\right) / 2\right)+S M_{a}$.

Os valores da segregação Mendeliana $\left(\mathbf{S M}_{\mathbf{a}}\right)$ foram calculados por meio da seguinte equação: $S M_{a}=Z_{n} \times C_{i j}$, em que: $\mathbf{S M}_{\mathbf{a}}$, são os contributos da segregação Mendeliana aleatória aos valores genéticos aditivos direto; $\mathbf{z}_{\mathbf{n}}$ são desvios aleatórios com distribuição normal; $\mathbf{C}_{\mathbf{i j}}$ são termos da decomposição 
de Cholesky da matriz G, correspondentes às componentes $\sigma_{\mathrm{a} 1}^{2}$, $\sigma_{\mathrm{ala} 2}$ e $\sigma_{\mathrm{a} 2}^{2}$, respectivamente. Os efeitos da segregação Mendeliana foram estimados independentes dos valores genéticos aditivos diretos dos animais. A decomposição de Cholesky da matriz de (co)variâncias genética (G) foi obtida por meio da função ROOT do procedimento Interactive Matrix Language (PROC IML) do SAS (SAS INSTITUTE, 2004).

Depois de criados rebanhos por meio de simulação, foram estimados valores genéticos (da população simulada) referentes a cada uma das características, a partir da análise uni-característica, $y=X \beta+Z a+e$, em que, $y$ : vetor de observações (peso final, ganho médio diário, avaliação de tipo e perímetro escrotal); $\boldsymbol{\beta}$ : vetor do efeito fixo (ano de nascimento); $\boldsymbol{a}$ : vetor do efeito genético aditivo; $\boldsymbol{X}$ : matriz de incidência que associa $\boldsymbol{\beta}$ comy; $\boldsymbol{Z}$ é $a$ matriz de incidência do efeito genético aditivo; e, $\boldsymbol{e}$ : vetor residual.
As estimativas dos valores genéticos para cada característica foram obtidas pelo método da Máxima Verossimilhança Restrita livre de Derivadas - DFREML, por meio do o programa MTDFREML, conforme descrito por Boldman et al. (1995). Para avaliar as tendências genéticas utilizou-se a regressão das médias anuais dos valores genéticos (aditivo) sobre o ano de nascimento do animal.

Com o objetivo de facilitar a compreensão sobre os índices utilizados neste estudo, foi elaborado o Tabela 3 , no qual são representados os tipos de índices, objetivo de seleção, índices e componentes dos índices. Convém destacar que o índice empírico (IE) refere-se ao índice atualmente utilizado para classificação de animais participantes das provas de ganho em peso, enquanto os demais índices de seleção (IS $\mathrm{PF}_{\mathrm{P}}$ e $\mathrm{IS}_{\mathrm{GMD}}$ ) têm sido criados e propostos com o intuito de avaliar a eficiência de utilização dos atuais índices empíricos.

Tabela 3. Tipos de índices, objetivo de seleção, índices e componentes dos índices

\begin{tabular}{lccl}
\hline Tipo & Objetivo de seleção & Índice & \multicolumn{1}{c}{ Componentes } \\
\hline \multirow{2}{*}{ IE } & - & I & PF + GMD + EPMURAS \\
& & II & PF + GMD + EPMURAS + PE \\
IS & & I & PF + GMD + EPMURAS \\
\multirow{2}{*}{ IS } & Peso final & II & PF + GMD + EPMURAS + PE \\
& \multirow{2}{*}{ Ganho médio diário } & I & PF + GMD + EPMURAS \\
& & II & PF + GMD + EPMURAS + PE
\end{tabular}

$\mathrm{IE}=$ índice empírico; $\mathrm{IS}_{\mathrm{PF}}=$ índice de seleção com objetivo peso final; $\mathrm{IS}_{\mathrm{GMD}}=$ índice de seleção com objetivo ganho médio diário; $\mathrm{PF}=$ peso final; $\mathrm{GMD}=$ ganho médio diário; EPMURAS = estrutura, precocidade, musculosidade, umbigo, racial, aprumos e sexual; $\mathrm{PE}=$ perímetro escrotal; AOL = área de olho de lombo; EGS = espessura de gordura de subcutânea

\section{RESULTADOS E DISCUSSÃO}

Os resultados desta pesquisa indicam que a utilização de touros jovens em provas de ganho em peso em regime de confinamento, quando classificados pelo índice II, o qual avalia as características de crescimento, avaliação de tipo e perímetro escrotal, independente do 
Rev. Bras. Saúde Prod. Anim., Salvador, v.13, n.3, p.669-681 jul./set., 2012 http://www.rbspa.ufba.br ISSN 15199940

objetivo de seleção, propicia às suas progênies um ganho genético maior do que a progênie de touros avaliados com os índices I (Tabela 4).

Os valores apresentados na Tabela 3 mostram que quanto maior o número de características avaliadas maior será a resposta à seleção, dessa forma, a utilização dos índices permite a predição de ganhos simultâneos a todas as características estudadas.

Tabela 4. Objetivo de seleção, pesos das características selecionadas, acurácia e resposta à seleção

\begin{tabular}{|c|c|c|c|c|c|c|c|}
\hline \multirow{2}{*}{$\begin{array}{l}\text { Objetivo de } \\
\text { seleção }\end{array}$} & \multirow{2}{*}{ Índice } & \multicolumn{4}{|c|}{ Característica } & \multirow{2}{*}{ Acurácia } & \multirow{2}{*}{$\begin{array}{c}\text { Resposta à seleção } \\
(\mathrm{kg} / \mathrm{ano})\end{array}$} \\
\hline & & $\mathrm{PF}$ & GMD & EPMURAS & $\mathrm{PE}$ & & \\
\hline \multirow{2}{*}{ EMPÍRICO ${ }^{1}$} & I & 0,40 & 0,40 & 0,20 & - & - & - \\
\hline & II & 0,35 & 0,35 & 0,20 & 0,10 & - & - \\
\hline \multirow{2}{*}{ PF } & I & 0,56 & 1,71 & 0,63 & - & 0,78 & 5,40 \\
\hline & II & 0,35 & 1,69 & 1,42 & 2,27 & 0,81 & 5,57 \\
\hline \multirow{2}{*}{ GMD } & I & 0,003 & 6,528 & 0,085 & - & 0,80 & 0,07 \\
\hline & II & 0,058 & 6,532 & $-0,128$ & $-0,612$ & 0,84 & 0,13 \\
\hline
\end{tabular}

${ }^{1}$ Josahkian et al. (2009)

$\mathrm{PF}=$ peso final; GMD = ganho médio diário; EPMURAS = avaliação de tipo; e PE = perímetro escrotal

A resposta à seleção, mediante a utilização desses índices (I, II), apresentou valores similares para cada objetivo de seleção, o que corrobora os trabalhos de Safus et al. (2006). Os relatos desses autores mostraram que os resultados obtidos foram semelhantes, independente da quantidade de informações que compõem o índice.

É importante destacar que o índice de seleção não escolhe os extremos, por possuir um limite de variância, sem permitir assim, extrapolar o valor definido. Esse aspecto é importante para o melhoramento genético animal, visto permitir a seleção dos melhores animais para todas as características simultaneamente.

Ainda na Tabela 4, pode ser visto que a utilização do índice de seleção, relacionado ao peso final (IS $S_{P F}$ ), promoverá uma resposta superior, quando comparado ao índice de seleção cujo objetivo foi ganho médio diário ( $\mathrm{IS}_{\mathrm{GMD}}$ ). Esses resultados são decorrentes de uma alta estimativa para variância de peso final, o que torna necessário salientar que quanto maior a variabilidade genética maiores serão as mudanças genéticas.

Para a característica peso final (Figura 1), o índice I apresentou maiores estimativas dos valores genéticos quando utilizou

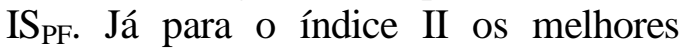
resultados foram obtidos a partir do $\mathrm{IS}_{\mathrm{GMD}}$, embora, suas tendências tenham apresentado evoluções similares.

No índice II, a inclusão da característica perímetro escrotal pode ter favorecido o ganho genético para os animais selecionados pelo IS $_{\mathrm{GMD}}$, devido às relações genéticas entre as características inclusas nesse índice. Da mesma forma, a estimativa de correlação genética alta e positiva $(0,73)$ entre peso final $\mathrm{e}$ perímetro escrotal, ponderou os pesos desses critérios de seleção, o que diminuiu a participação do peso final no contributo geral do índice. Essa relação genética promoveu decréscimo do ganho genético do peso final quando avaliado pelo $\mathrm{IS}_{\mathrm{GMD}}$. 

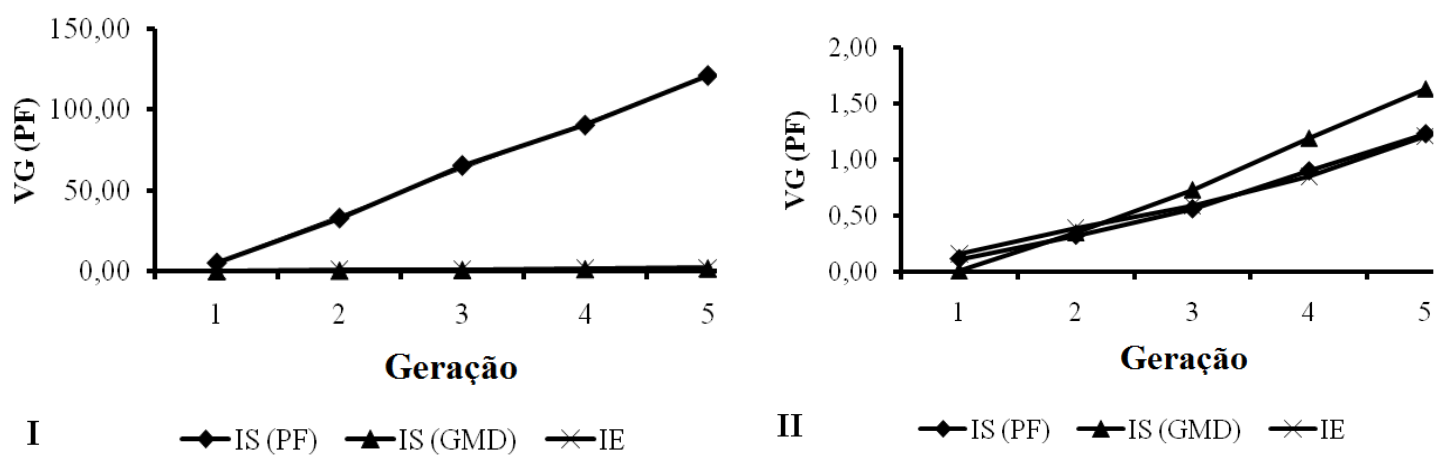

IS $(\mathrm{PF})=$ Índice de seleção para peso final; ID (GMD) = Índice de seleção para ganho em peso; $\mathrm{IE}=$ Índice empírico

Figura 1. Tendência genética do valor genéticos dos animais para peso final nos índices I e II

Os melhores resultados para o ganho médio diário foram verificados ao utilizar qualquer um dos índices, quando o objetivo de seleção foi ganho médio diário (Figura 2), porém, apresentaram baixas estimativas dos valores genéticos para ganho médio diário, devido à pequena variância existente para essa característica, o contributo para o melhoramento genético é pequeno. Isso, talvez, se deva à utilização de animais pré-selecionados, o que implica diretamente na redução desta variância.
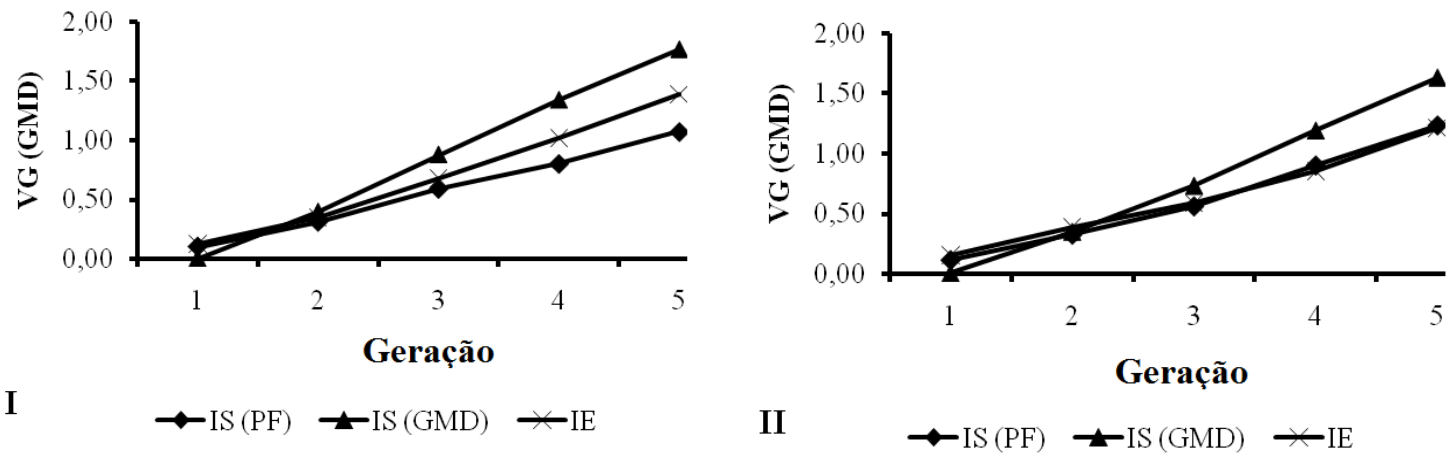

IS $(\mathrm{PF})=$ Índice de seleção para peso final; ID (GMD) = Índice de seleção para ganho em peso; $\mathrm{IE}=$ Índice empírico

Figura 2. Tendência genética do valor genéticos dos animais para ganho em peso médio diário nos índices I e II

A utilização do $\mathrm{IS}_{\mathrm{GMD}}$ não favoreceu o progresso genético para as demais características como pode ser verificado nas Figuras 1, 2, 3 e 4. Isso pode ser explicado pelas baixas correlações genéticas existentes entre ganho médio diário e as outras características inclusas nos índices. As estimativas de correlações apresentaram magnitudes de 0,24, 0,23 e -0,11 para GMD e PF, GMD e EMPURAS e GMD e PE, respectivamente. 
Na Figura 3, observa-se que a utilização do índice empírico (IE) foi semelhante ao $\mathrm{IS}_{\mathrm{PF}}$, cujas tendências apresentam uma sobreposição aos índices I e II. No entanto, essas tendências foram superiores, quando comparadas com a seleção realizada por meio do $\mathrm{IS}_{\mathrm{GMD}}$. A correlação genética entre peso final e avaliação de tipo possui estimativas moderadas a altas $(0,58)$, o que justifica dessa forma $\mathrm{o}$ maior valor genético quando se utiliza $\mathrm{IS}_{\mathrm{PF}}$. A estimativa de correlação genética entre ganho médio diário e avaliação de tipo foi de baixa magnitude $(0,28)$. Pires et al. (2010), ao estudarem características morfológicas e de crescimento de bovinos da raça Nelore, também evidenciaram estimativas de correlação genética positiva entre peso e características morfológicas de estrutura corporal, precocidade de terminação e musculosidade.
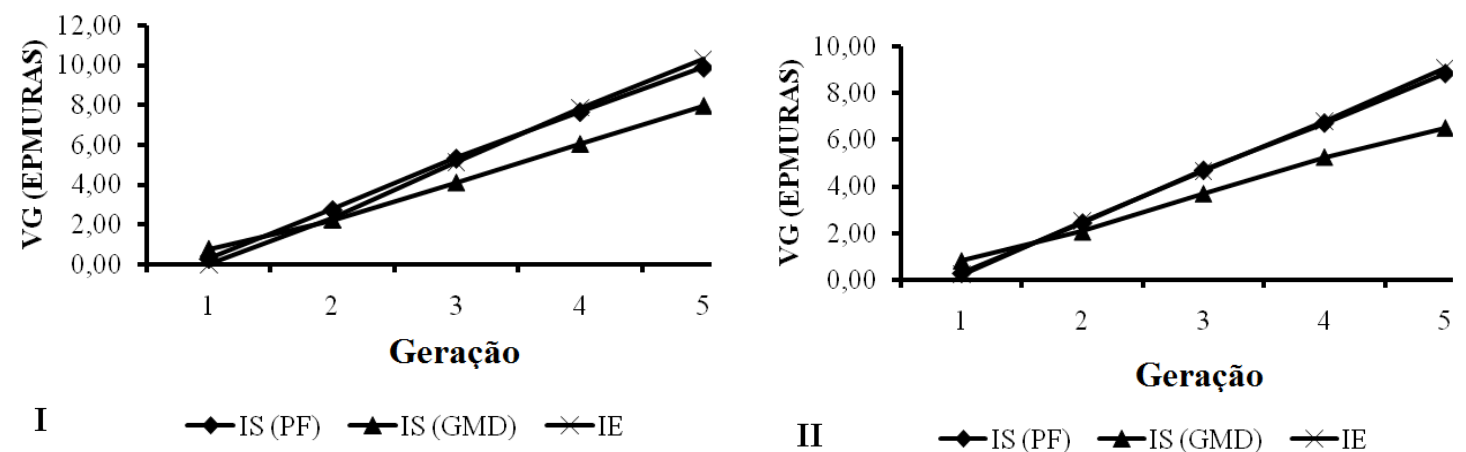

IS $(\mathrm{PF})=$ Índice de seleção para peso final; ID $(\mathrm{GMD})$ = Índice de seleção para ganho em peso; IE = Índice empírico

Figura 3. Tendência genética do valor genéticos dos animais para EPMURAS nos índices I e II

A tendência genética para a característica perímetro escrotal (Figura 4) apresentou altas estimativas de valor genético, quando se utilizou $\mathrm{IS}_{\mathrm{PF}}$. Com o índice I verificaram-se tendências similares, independente do objetivo de seleção. Já para o índice II, as regressões obtidas por meio do $\mathrm{IS}_{\mathrm{PF}}$ apresentaram estimativas aproximadas ao IE e superiores às tendências observadas ao utilizar IS $_{\mathrm{GMD}}$. A utilização de peso final como objetivo de seleção promoveu ganhos em todas as características, independente do índice utilizado (I e II), o que torna importante lembrar que foram apresentadas tendências similares para a característica avaliação de tipo, ao se utilizar IE e IS $\mathrm{PF}_{\mathrm{PF}}$
Observou-se que ao longo dos anos, os produtores se utilizaram de índices empíricos. Assim, esses resultados foram importantes ao demonstrar que os três índices (IE, IS $\mathrm{S}_{\mathrm{PF}}$ e $\mathrm{IS}_{\mathrm{GMD}}$ ) apresentaram tendências similares e promoveram melhorias em todas as características, embora com intensidades diferentes para cada índice.

Entre as provas zootécnicas que avaliam o desempenho dos indivíduos da raça Nelore, as provas de ganho em peso (PGPs) se constituem importante ferramenta na avaliação dos animais. Dessa forma, os animais participantes dessas provas têm sido selecionados de acordo com as principais características de importância econômica. A prova de ganho em peso é considerada uma 
ferramenta de seleção eficiente, porém os índices de classificação, atualmente utilizados, foram criados e propostos de maneira empírica, sem serem, portanto obtidos, de conceitos preconizados ou disponíveis na teoria de índices de seleção.

Embora, neste estudo, não se tenha estimado os valores econômicos das características analisadas, a ponderação empírica, em peso final e ganho médio diário, foi essencial porque são as características de maior impacto econômico para os sistemas de produção de bovinos de corte. Os resultados deste estudo demonstraram que a utilização de índices de seleção é a melhor maneira de ponderar um conjunto de características de interesse.
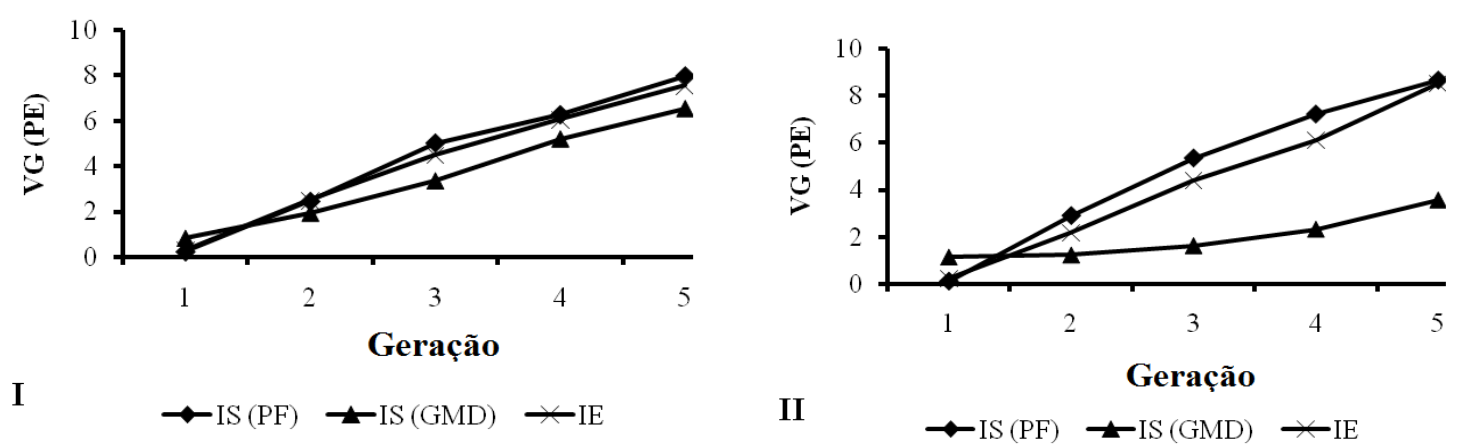

IS (PF) = Índice de seleção para peso final; ID (GMD) = Índice de seleção para ganho em peso; IE = Índice empírico

Figura 4. Tendência genética do valor genéticos dos animais para perímetro escrotal nos índices I e II

Ao se estudar os índices empíricos e de seleção, observou-se que o índice, cujo objetivo foi o peso final e que leva em conta as características peso final, ganho médio diário, avaliação de tipo, perímetro escrotal e ganho médio diário, promoveu maiores predições dos valores genéticos. A escolha dos critérios de seleção pode variar conforme a decisão e perfil dos produtores rurais

Recomenda-se então, a utilização dos índices de seleção na classificação dos animais participantes das PGPs, de forma a permitir assim a obtenção de ganhos simultâneos em todas as características avaliadas.

Os índices de seleção foram eficazes na escolha dos melhores animais para um conjunto de características, simultaneamente. Entretanto, são necessários novos estudos que avaliem e discriminem o valor econômico de todas as características que impactam economicamente os sistemas de produção de bovinos de corte no Brasil.

\section{REFERÊNCIAS}

BETT, R.C.; KOSGEY, I.S.; BEBE, B.O.; KAHI, A.K. Genetic improvement of the Kenya Dual Purpose Goat: Influence of economic values and prospects for a practical breeding programme. Tropical Science, v.47, n.3, p.105-119, 2007. 
BOLDMAN, K.G.; KRIESE, L.A.; VAN VLECK, L.D.; VAN TASSEL, C.P.; KACHMAN, S.D. A manual for use of MTDFREML: a set of programs to obtain estimates of variances and covariances. Beltsville: Department of Agriculture, Agricultural Research Service, 1995.

CHEN, J.; WANG, Y.; ZHANG, Y.; SUN, D.; ZHANG, Y. Estimation of economic values for production and functional traits in Chinese Holstein. Journal of Animal and Veterinary Advances, v.8, n.11, p.2125-2132, 2009.

CLÉMENT, V.; BIBÉ, B.; VERRIER, E.; ELSEN, J. M.; MANFREDI, E.; BOUIX, J.; HANOCQ, E. Simulation analysis to test the influence of model adequacy and data structure on the estimation of genetic parameters for traits with direct and maternal effects. Genetics Selection Evolution, v.33, n.4, p.369-395, 2001.

CUNNINGHAM, E.P.; TAUEBERT, $\mathrm{H}$. Measuring the effect of change in selection indices. Journal of Dairy Science, v.92, p.6192-6196, 2009.

DUBEUF, J.P.; BOYAZOGLU, J. An international panorama of goat selection and breeds. Livestock Science, v.120, p.225-231, 2009.

HAZEL, L.N. The genetic basis for constructing selection indexes.

Genetics, v.28, p.476-490, 1943.

JOSAHKIAN, L.A.; LUCAS, C.H.; MACHADO, C.H.C. Manual do serviço de registro genealógico das raças zebuínas e PMGZ: manual de operação. Uberaba: Associação Brasileira dos Criadores de Zebu, 2009. 190p.
KRUPOVÁ, Z.; ORAVCOVÁ, M.; KRUPA, E.; PEŠKOVIČOVÁ, D. Methods for calculating economic weights of important traits in sheep. Slovak Journal of Animal Science, v.41, n.1, p.24-29, 2008.

KRUPOVÁ, Z.; HUBA, J.; DANO, J.; KRUPA, E.; ORAVCOVÁ, M.; PEŠKOVIČOVÁ, D. Economic weights of production and functional traits ini dairy cattle under a direct subsidy regime. Czech Journal of Animal Science, v.54, n.6, p.249-259, 2009.

LAMBE, N.R.; BÜNGER, L.; BISHOP, S.C.; SIMM, G.; CONINGTON, J.The effects of selection indices for sustainable hill sheep production on carcass composition and muscularity of lambs, measured using X-ray computed tomography. Animal, v.2, n.1, p.27-35, 2008.

LÔBO, R.N.B.; FACÓ, O.; LÔBO, A.M.B.O.; VILLELA, L.C.V. Brazilian goat breeding programs. Small

Ruminant Research, v.89, p.149-154, 2010.

MAGHSOUDI, A.; TORSHIZI, R.V.; JAHANSHAHI, A.S. Estimates of (co)variance components for productive and composite reproductive traits in Iranian Cashmere goats. Livestock Science, v.126, p.162-167, 2009.

MARTINS, G.A.; MADALENA, F.E.; BRUSCHI, J.H.; COSTA, J.L.; MONTEIRO, J.B.N. Objetivos econômicos da seleção de bovinos de leite para fazenda demonstrativa na Zona da Mata de Minas Gerais. Revista Brasileira de Zootecnia, v.32, n.02, p.304-314, 2003. 
Rev. Bras. Saúde Prod. Anim., Salvador, v.13, n.3, p.669-681 jul./set., 2012 http://www.rbspa.ufba.br ISSN 15199940

PEDROSA, V.B.; ELER, J.P.; FERRAS, J.B.S.; SILVA, J.A. II V.; RIBEIRO, S.; SILVA, M.R.; PINTO, L.F.B. Parâmetros genéticos do peso adulto e características de desenvolvimento ponderalna raça Nelore. Revista Brasileira de Saúde e Produção Animal [Online], v.11, n.1, p 104-113, 2010.

PIRES, B.C.; FARIA,C.U.; VIU, M.A.O.; TERRA, J.P.; LOPES, D.T.; MAGNABOSCO, C.U.; LÔBO, R.B. Modelos bayesianos de limiar e linear na estimação de parâmetros genéticos para características morfológicas de bovinos da raça Nelore. Revista Brasileira de Saúde e Produção Animal [Online], v.11, n.3, p.651-661, 2010.

QUEIROZ, S.A.; PELICIONI, L.C.; SILVA, B.F.; SESANA, J.C.; MARTINS, M.I.E.G.; SANCHES, A. Índices de seleção para um rebanho Caracu de duplo propósito. Revista Brasileira de Zootecnia, v.34, n.3, p.827-37, 2005.

SAFUS, P.; PŘIBYL, J.; VESELÁ, Z.; VOSTRÝ, L.; ŠTÍPKOVÁ, M.; STÁDNÍK, L. Selection indexes for bulls of beef cattle. Czech Journal Animal Science, v. 51, n. 7, p.285-298, 2006.

\section{SAS INSTITUTE. SAS onlineDOC ${ }^{\circledR}$} 9.1.3. Cary, NC. 2004.
TABBAA, M.J.; AL-ATIYAT, R. Breeding objectives, selection criteria and factors influencing them for goat breeds in Jordan. Small Ruminant Research, v.84, p.8-15, 2009.

TOZER, P.R.; STOKEST, J.R.

Producer breeding objectives and optimal sire selection. Journal of Dairy Science, v.85, p.3518-3525, 2002.

WOLFOVÁ, M.; WOLF, J.; PRIBYL, J. Impact of milk pricing system on the economic response to selection on milk components. Journal of Animal Breeding and Genetic, v.124, p.192200, 2007a.

WOLFOVÁ, M.; WOLF, J.; KVAPILÍK, J.; KICA, J. Selection for profit in cattle: i. economic weights for purebred dairy in the CzechRepublic.

Journal of Dairy Science, v.90. p.2442-2455, $2007 b$.

WOLFOVÁ, M.; WOLF, J.; KVAPILÍK, J.; KICA, J. Selection for profit in cattle: ii. economic weights for purebred dairy and beef sires in crossbreeding systems. Journal of Dairy Science, v.90. p.2456-2467, 2007c.

ZHANG, C.Y.; CHEN, S.L.; LI, X.; XU, D.Q.; ZHANG, Y.; YANG, L.G. Genetic and phenotypic parameter estimates for reproduction traits $\mathrm{n}$ the Boer dam. Livestock Science, v.125, p.60-65, 2009.

Data de recebimento: 07/09/2011

Data de aprovação: 08/05/2012 